\title{
Inactivated probiotic Bacillus coagulans GBI-30 induces complex immune activating, anti-inflammatory, and regenerative markers in vitro
}

\section{Gitte S Jensen' \\ Howard A Cash ${ }^{2}$ \\ Sean Farmer ${ }^{2}$ \\ David Keller ${ }^{2}$}

'NIS Labs, Esplanade, Klamath Falls, OR, USA, ${ }^{2}$ Ganeden Biotech Inc., Landerbrook Drive Suite, Mayfield Heights, OH, USA
Correspondence: Gitte S Jensen NIS Labs, I 437 Esplanade, Klamath Falls, OR 9760I, USA

$\mathrm{Tel}+\mathrm{I} 5418840112$

Email gitte@nislabs.com
This article was published in the following Dove Press journal:

Journal of Inflammation Research

7 August 2017

Number of times this article has been viewed

Objective: The aim of this study was to document the immune activating and anti-inflammatory effects of inactivated probiotic Bacillus coagulans GBI-30, 6086 (Staimune ${ }^{\mathrm{TM}}$ ) cells on human immune cells in vitro.

Methods: In vitro cultures of human peripheral blood mononuclear cells (PBMC) from healthy blood donors were treated with inactivated B. coagulans GBI-30, 6086 cells for 24 hours. After incubation, the PBMC were stained with fluorochrome-labeled monoclonal antibodies for CD3, CD56, and CD69 to monitor cellular activation by flow cytometry. The culture supernatants were tested for cytokine profile using a 27-plex Luminex array, including pro- and anti-inflammatory cytokines, chemokines, and growth factors.

Results: Inactivated B. coagulans GBI-30, 6086 cells induced the CD69 early activation marker on $\mathrm{CD}^{+}{ }^{+} \mathrm{CD} 56^{-} \mathrm{T}$ lymphocytes, $\mathrm{CD} 3{ }^{+} \mathrm{CD} 56^{+} \mathrm{NKT}$ cells, $\mathrm{CD} 3^{-} \mathrm{CD} 56^{+} \mathrm{NK}$ cells, and also some cells within the $\mathrm{CD}^{-}{ }^{-} \mathrm{CD} 56^{-}$non-T non-NK cell subset. Culture supernatants showed robust increases in the immune-activating cytokines IL-1 $\beta$, IL-6, IL-17A, and TNF- $\alpha$. IFN- $\gamma$ levels were increased, along with three chemokines, MCP-1, MIP-1 $\alpha$, and MIP-1 $\beta$. The two antiinflammatory cytokines IL-1ra and IL-10 showed increases, as well as the G-CSF growth factor involved in repair and stem cell biology. In contrast, GM-CSF levels showed a mild decrease, showing a highly selective growth factor response.

Conclusion: The inactivated $B$. coagulans GBI-30, 6086 cells activated human immune cells and altered the production of both immune activating and anti-inflammatory cytokines and chemokines. Of special importance is the novel demonstration of a selective upregulation of the G-CSF growth factor involved in postinjury and postinflammation repair and regeneration. This suggests that important immunogenic cell wall components, such as lipoteichoic acid, are undamaged after the inactivation and retain the complex beneficial biological activities previously demonstrated for the cell walls from live B. coagulans GBI-30, $6086\left(\right.$ GanedenBC $\left.^{30}\right)$ probiotic bacteria.

Keywords: anti-viral, anti-inflammatory, cytokines, growth factors, lipoteichoic acid, inactivated bacillus coagulans GBI-30, 6086, Staimune

\section{Plain language summary}

This study was done to test a new consumable health product, made by inactivating a specific strain of beneficial probiotic gut bacteria. We have previously shown that this strain of gut bacteria activates human immune cells and helps mature certain immune cells that are of importance for detecting foreign antigens. The inactivating process allows the bacterium to be used in broader applications, such as foods, where a living bacterium could spoil the food or give it a very limited 
shelf life. It was important to show that the inactivated bacteria had similar properties to the live bacteria.

To test this, we used blood samples from healthy humans and isolated a part of the white blood cells that include immune cells and stem cells. The blood samples contained the same types of cells as in the blood circulation in our intestinal walls, where antigens from the gut are presented to immune cells. We cultured the cells with the inactivated bacterial cells for 24 hours. We examined the immune cells for their activation status. We tested the liquid culture medium for secreted biomarkers.

We found that the inactivated bacterial cells had similar effects as the live ones with respect to immune activation and antiinflammatory effects. We also found novel effects that showed that the human cells secreted a growth factor important for tissue repair after trauma and injury.

\section{Introduction}

The gut microbial community, "gut microbiome", has a vast impact on the health of the human host. ${ }^{1,2}$ An integral collaboration exists between microbial forms colonizing the gut and our immune function, metabolism, and brain function. ${ }^{3}$ The interaction between gut microbes and host cells and tissue takes place in several ways, including via bacterial cell wall components and secreted metabolites. The most immediate and direct interaction between microbes and host cells involves the outer layers of bacterial cell wall components engaging with receptors on immune cells, such as dendritic cells directly sampling antigens in the gut lumen. This interaction presents different types of bacterial cell wall components of Gram-positive versus Gram-negative bacteria. Gram-negative bacteria present lipopolysaccharides (LPSs), which are recognized by toll-like receptor-4 (TLR-4), ${ }^{4}$ whereas the outer cell walls of Gram-positive bacteria present teichoic acid and lipoteichoic acid to immune cells, recognized by TLR-2. ${ }^{5}$ For many pathogenic bacteria, lipoteichoic acid is associated with virulence, ${ }^{6}$ whereas lipoteichoic acid from beneficial probiotic bacteria trigger complex beneficial immune modulation. Lipoteichoic acid has been widely used as a model TLR-2 ligand to explore a wide variety of immune activating mechanisms at the cellular and molecular level, and interestingly has proven to exert both proinflammatory ${ }^{7,8}$ and anti-inflammatory ${ }^{9,10}$ activities in vitro. The structural complexity of lipoteichoic acid is said to impact the host immune response. The chemical composition of this acid differs between microbes and between strains of similar microbes. This is of high importance in triggering diverse effects on host cells and is likely one of the key factors in the highly selective immune-modulating effects induced by different microbial strains.

The consumption of beneficial probiotic bacteria is associated with a range of health benefits tied to inflammation regulation, such as gastrointestinal disease, ${ }^{11}$ respiratory tract infections, ${ }^{12}$ neuro-immune and neuropsychiatric disorders, ${ }^{13}$ satiety and psychosocial behavior in obese individuals, ${ }^{14}$ and alleviation of symptoms of anxiety and depression, ${ }^{15}$ as a result of the extensive communication between the gastrointestinal and central nervous systems, also referred to as the "gut-brain axis". ${ }^{16}$ While the consumption of probiotic bacteria is considered highly safe, there are many useful applications for inactivated probiotic strains, such as increased shelf life, as well as usefulness in many types of food products where metabolically active, living bacteria may spoil the appearance of the food. Inactivated probiotic bacteria also have a use in specific clinical situations involving immune-compromised individuals where there could potentially be a risk of translocation of gut bacteria into the bloodstream. Inactivated probiotic bacteria can be produced, for example by heating, leaving the outer bacterial cell wall as the main mechanism of interaction with host immune cells. Heatkilled Lactobacillus plantarum L-137 (HK L-137) has been widely studied over the past decades for its effects in rodents and humans. Animal studies have shown that consumption of HK L-137 offers protection against influenza virus infection, associated with increased production of interferons, suggesting a general support of antiviral immune defense activity. ${ }^{17}$ Clinical trials have shown that the daily intake of HK L-137 supports a healthy immune function, including enhanced acquired immune responses and TH1-related immune function, ${ }^{18}$ and reduced incidence of upper respiratory tract infections. ${ }^{19}$ Consumption was also associated with improved oral health. ${ }^{20}$ The heat-killed bacterial cells are also known to exert antiallergic ${ }^{21}$ and antitumor $^{22}$ effects, in part due to the potent induction of IL-12 and interferons ${ }^{23}$ by lipoteichoic acids on the bacterial cell wall surfaces. Furthermore, the L-137 strain has higher levels of lipoteichoic acids exposed on the surface, with higher amounts of alanine, than the closely related L. plantarum JCM1149 strain; ${ }^{24}$ this correlates with the higher induction of IL-12 by the L-137 strain than by the JCM1149 strain. $^{25}$

Another group of lactic acid-producing probiotic bacteria includes several unique strains of the spore-forming B. coagulans (previously classified as L. sporogenes). 
The teichoic acid from B. coagulans walls has a higher lipid content than most Gram-positive bacteria, and is a glycerophosphate polymer substituted with two neutral sugars, glucose and galactose. It is unique in lacking amino acid substituents, otherwise considered a characteristic of teichoic acids. ${ }^{26}$

Cell walls from the live B. coagulans GBI-30, 6086 strain $\left(\right.$ GanedenBC $\left.{ }^{30}\right)$ have demonstrated immune modulating and anti-inflammatory effects in vitro. ${ }^{27}$ Our team previously showed that immune-modulating effects of the BC30 strain were associated both with the cell wall fraction and with the metabolites produced by the live bacteria in vitro. ${ }^{28}$ The probiotic strain was further shown to prolong the survival and reduce symptoms in mice infected with Clostridium difficile. ${ }^{29,30}$ Clinical studies showed that consuming BC30 helped alter the gut microbiome by increasing the numbers of beneficial bacteria, ${ }^{31}$ and ex vivo testing of blood from elderly humans who had consumed BC 30 for 28 days showed increased anti-inflammatory cytokine responses. ${ }^{32}$ Results from a recent clinical trial suggest that the consumption of $\mathrm{BC} 30$ supports exercise performance and helps reduce exercise-induced muscle damage. ${ }^{33}$

Recently, inactivated B. coagulans GBI-30, 6086 cells have been produced for oral consumption. The work presented here was undertaken to document whether the immune activating and anti-inflammatory properties associated with the cell walls of the live $\mathrm{BC} 30$ bacteria were protected in the inactivated product. An important focus for this work was to document the biological activities of the inactivated $B$. coagulans GBI-30 bacterial cells when presented to human immune cells in a cell culture system that allows cross-talk between antigen-presenting monocytes and dendritic cells with lymphocytes and natural killer cells, and thus mimics events in the gut mucosal immune tissue.

\section{Materials and methods Reagents}

Phosphate-buffered saline, Roswell Park Memorial Institute 1640 (RPMI-1640) medium, penicillin-streptomycin $100 \times$, IL-2, and LPS were purchased from Sigma-Aldrich Co. (St. Louis, MO, USA). CD69 fluorescein isothiocyanate, CD56 phycoerythrin, CD3 peridinin chlorophyll protein, CD25 brilliant violet 421 and heparin Vacutainer vials were purchased from BD Biosciences (Franklin Lakes, NJ, USA). The Bio-Plex Pro ${ }^{\mathrm{TM}}$ human cytokine 27-Plex was purchased from Bio-Rad Laboratories Inc. (Hercules, CA, USA).

\section{Inactivated B. coagulans GBI-30 (Staimune ${ }^{\mathrm{TM}}$ )}

Inactivated B. coagulans GBI-30, 6086 cells were provided by Ganeden Inc. (Mayfield Heights, OH, USA). Bacterial cell numbers were 15 billion $\left(1.5 \times 10^{10}\right) \mathrm{CFU}$ per gram prior to inactivation. Inactivated bacteria were diluted in physiological saline and added to human peripheral blood mononuclear cell cultures at doses from $0.78 \times 10^{6}-100 \times 10^{6}$ inactivated cells/mL cell culture.

\section{Peripheral blood mononuclear cell cultures}

Healthy human volunteers between the age of 50 and 60 years served as blood donors upon informed consent, as approved by the Sky Lakes Medical Center Institutional Review Board (FWA 2603). Freshly drawn peripheral venous blood samples in sodium heparin were layered onto Lympholyte-Poly and centrifuged for 35 minutes at $450 \mathrm{~g}$. The upper peripheral blood mononuclear cells (PBMC)-rich interface was harvested using sterile transfer pipettes into new vials and washed twice with $10 \mathrm{~mL}$ PBS, without calcium or magnesium, by centrifugation for 10 minutes. The cells were resuspended into RPMI 1640 with $10 \%$ fetal bovine serum, L-glutamine, and antibiotics (penicillin and streptomycin) to a cell density of $10^{6} / \mathrm{mL}$. Triplicate cultures were established for each of the eight doses of inactivated GBI-30 tested. Untreated cell cultures (negative controls) were established in hexaplicate. Two sets of positive control cultures were established in triplicates, one set using LPS (10 ng/mL) and another set using IL-2 (100 IU/mL) to activate the immune cells by two different pathways.

\section{Cytokine testing}

Supernatants were harvested from the human immune cell 24-hour cultures, and the levels of 27 cytokines, chemokines, and growth factors were analyzed. Testing was performed on culture supernatants from cell cultures treated with the six higher doses of inactivated GBI-30. The following markers: IL-1 $\beta$, IL-1ra, IL-2, IL-4, IL-5, IL-6, IL-7, IL-8, IL-9, IL-10, IL-12 (p70), IL-13, IL-15, IL-17, eotaxin, basic FGF, G-CSF, GM-CSF, Interferon-gamma (IFN- $\gamma$ ), IP-10, MCP-1, MCAF, MIP-1 $\alpha$, MIP-1 $\beta$, PDGF-BB, RANTES, TNF- $\alpha$, and VEGF were quantified using Bio-Plex protein arrays (Bio-Rad Laboratories Inc.) and utilizing XMAP technology (Luminex, Austin, TX, USA).

\section{Statistical analysis}

Averages and SDs for each data set were calculated using Microsoft Excel. Statistical analysis was performed using 
the 2-tailed, independent $t$-test. Statistical significance was indicated when $P<0.05$ and a high level of significance when $P<0.01$.

\section{Results}

\section{Immune cell activation}

Immune cell activation by inactivated $B$. coagulans GBI-30 cells was determined by measuring cell-surface expression of the activation marker CD69. The gating on immune cells with different forward and side scatter properties allowed analysis of CD69 expression on lymphocytes versus monocytes/macrophages (Figure 1). Treatment of both cell types with inactivated GBI-30 cells for 24 hours resulted in activation across a broad dose range. The results for CD69 expression on lymphocytes showed that even at the lowest dose the CD69 expression was not returning to baseline, and suggests that much lower doses would still have been able to activate lymphocytes (Figure 1A). In contrast, the most robust and statistically significant activation of monocytes was in a narrower dose range, returning toward baseline at the lowest dose shown (Figure 1B).
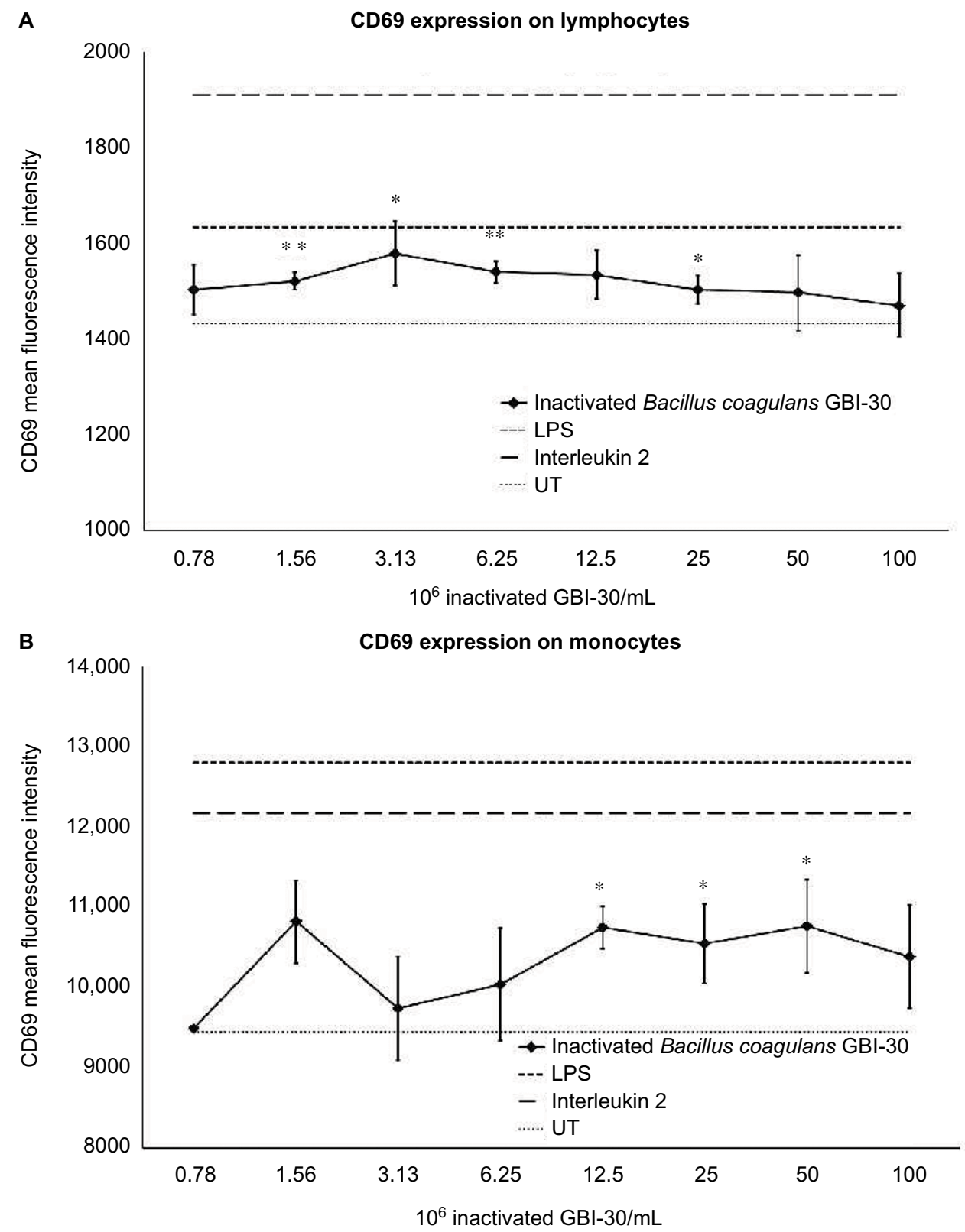

Figure I Expression of the CD69 cellular activation marker on lymphocytes and monocytes.

Notes: $* P<0.05 ; * * P<0.01$. CD69 expression on lymphocytes $(\mathbf{A})$ and monocytes $(\mathbf{B})$ in human PBMC cultures treated for 24 hours with serial dilutions of inactivated Bacillus coagulans GBI-30 cells. Mean fluorescence intensity for CD69 expression is shown. Data presented as mean \pm SD from triplicate cultures and represent one of three separate experiments using PBMC from three different healthy human donors. For lymphocytes, the mean \pm SD for the controls were: UT I,432 \pm 32 , LPS I,635 \pm 33 , and IL-2

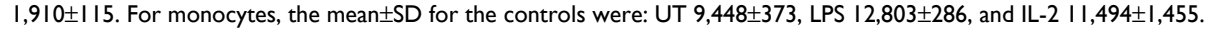

Abbreviations: LPS, lipopolysaccharide; PBMC, peripheral blood mononuclear cells; UT, untreated. 
The use of fluorescently labeled antibodies to CD3, CD56, and CD69 allowed the monitoring of changes to lymphocyte subsets, including $\mathrm{CD}^{+} \mathrm{T}$ lymphocytes, $\mathrm{CD}^{+}$ $\mathrm{CD}^{+} 6^{+}$Natural Killer T (NKT) cells, CD3 ${ }^{-} \mathrm{CD} 56^{+} \mathrm{NK}$ cells, and non-T non-NK lymphocytes. Treatment of PBMC cultures with inactivated GBI-30 cells for 24 hours led to the activation of T lymphocytes, NKT cells, NK cells, and non-T non-NK cells (Figure 2). T lymphocyte activation was seen across a broad dose range, and at the third-lowest dose $\left(3.13 \times 10^{6}\right.$ bacteria $\left./ \mathrm{mL}\right)$, the $\mathrm{CD} 69$ expression was as robust as for LPS-induced CD69 expression (Figure 2A). The T lymphocyte activation remained highly significant even at
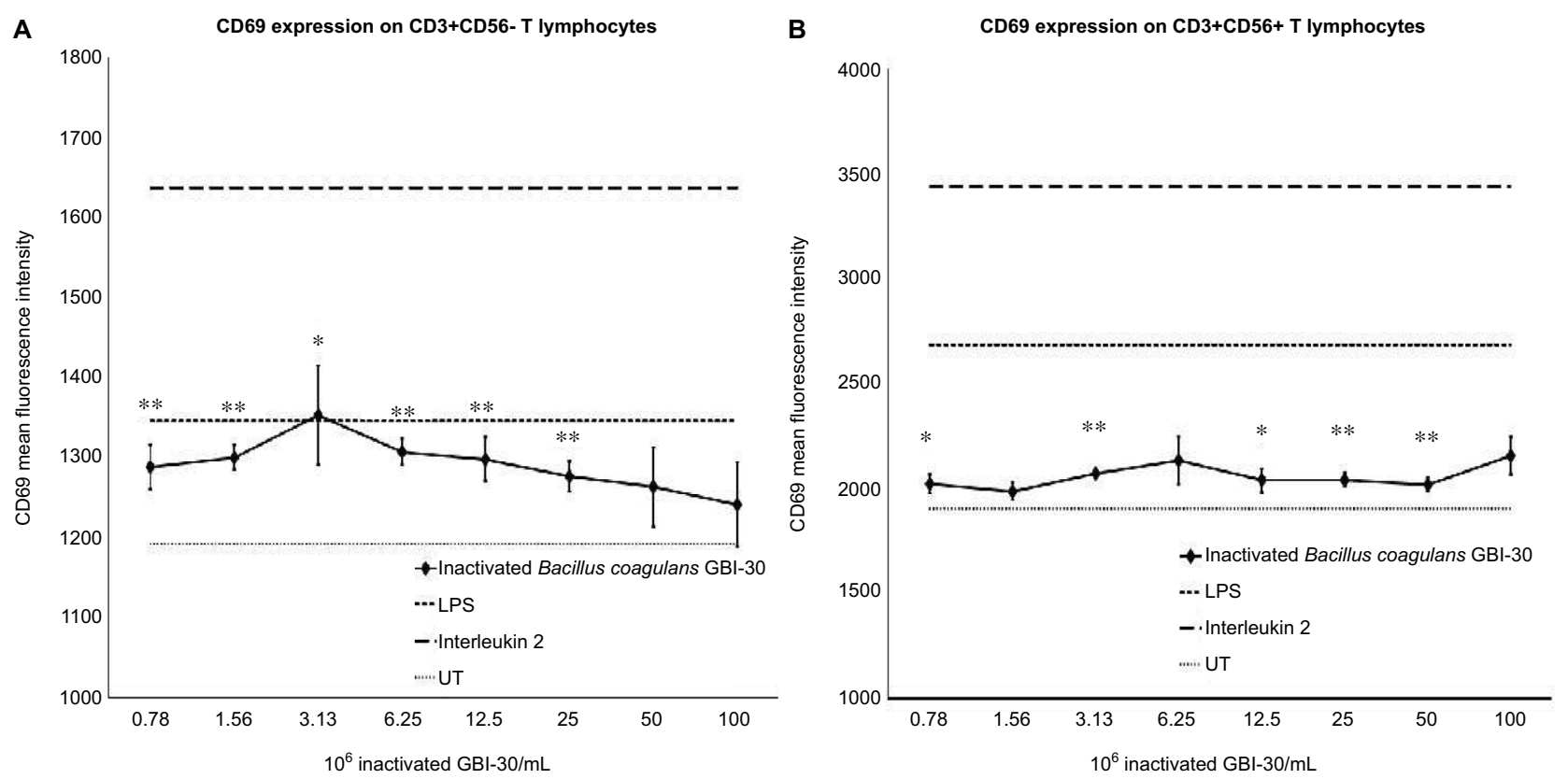

C

CD69 expression on CD3- CD56+ NK cells

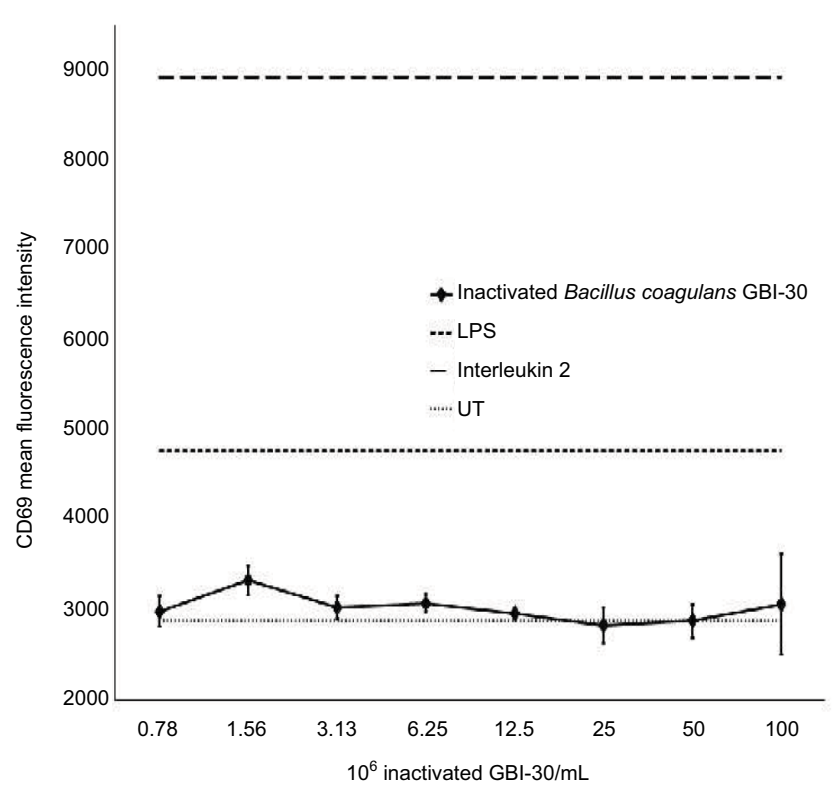

D

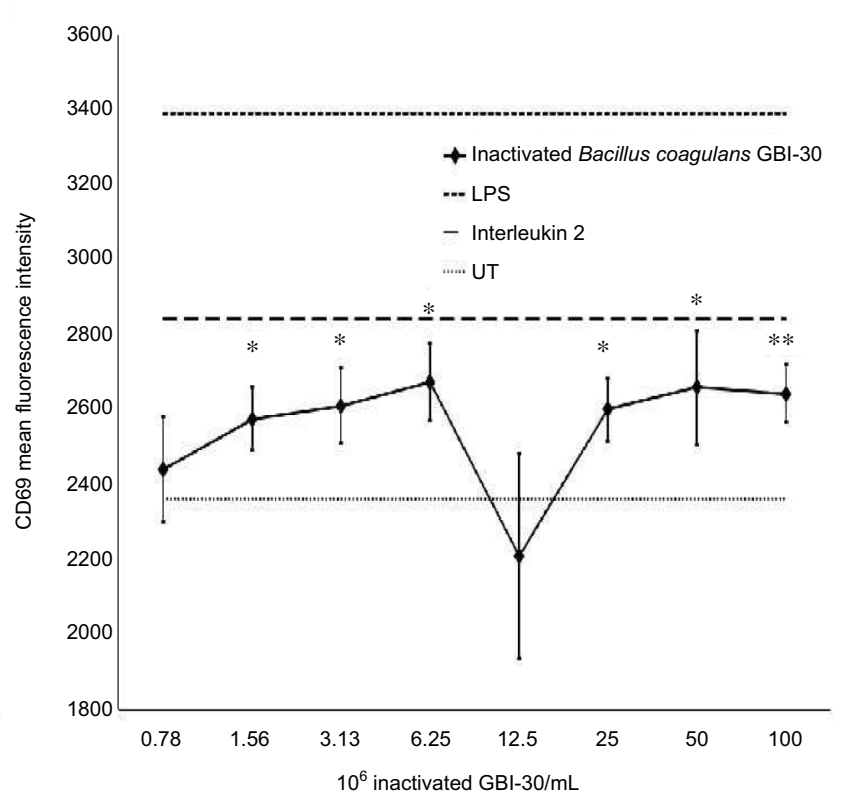

Figure 2 Expression of the CD69 cellular activation marker on immune cell subsets.

Notes: $* P<0.05 ; * * P<0.01$. CD69 expression on T lymphocytes (A), NKT cells (B), NK cells (C), and non-T non-NK cells (D) in human PBMC cultures treated for 24 hours with serial dilutions of inactivated Bacillus coagulans GBI-30 cells. Mean fluorescence intensity for CD69 expression is shown. Data presented as mean \pm SD from triplicate cultures and represent one of three separate experiments using PBMC from three different healthy human donors. For T lymphocytes, the mean \pm SD for the controls were: UT I, 192 \pm 23 , LPS I,346 \pm 20 , and IL-2 I,638 \pm 97 . For NKT cells, the mean \pm SD for the controls were: UT I,908 \pm 36 , LPS $2,687 \pm$ I 26, and IL-2 3,669 \pm 493 . For NK cells, the mean \pm SD for the controls were: UT 2,880 \pm 259 , LPS 4,770 \pm 503 , and IL-2 8,930 \pm I,6I3. For non-T non-NK cells, the mean \pm SD for the controls were: UT 2,364 \pm I 46, LPS $3,393 \pm 137$, and IL-2 2,935 \pm 184 .

Abbreviations: LPS, lipopolysaccharide; PBMC, peripheral blood mononuclear cells; UT, untreated. 
the lowest dose of inactivated GBI-30. Activation of NKT cells was also observed across the entire dose range, and at some doses, the activation was highly significant, compared to untreated control cultures (Figure 2B). NK cell activation was most robust at the lower doses and less prominent at higher doses (Figure 2C). The CD69 expression in the non-T non-NK lymphocyte population was seen for a broad dose range as well, returning to baseline at the lowest dose tested (Figure 2D). LPS was used as a positive control $(10 \mathrm{ng} / \mathrm{mL})$ and resulted in an increase in CD69 expression on all cell types. IL-2 was used as a second positive control (100 IU/ $\mathrm{mL}$ ) and also showed an increase in CD69 on all cell types.

Please note that occasionally, a large variation in CD69 expression was seen within one set of triplicate cultures, as reflected by large error bars, and in some cases, an average response in the triplicate set appears out of line with the overall dose response. We suggest that this is due to the nature of the test product, where the inactivated bacterial cells may clump within a culture well instead of dispersing, thus not providing optimal interaction between bacterial cells and PBMC within those wells. An example is the very low CD69 expression on non-T non-NK lymphocytes at the dose of $12.5 \times 10^{6}$ inactivated GBI-30/mL, where only one of the three triplicate culture wells showed the CD69 expression level comparable to the dose above and the dose below (Figure 2D).

\section{Immune-activating cytokines $B$. coagulans} Supernatants from the PBMC cultures exposed to inactivated B. coagulans GBI-30 cells for 24 hours were simultaneously assayed for the levels of 27 different cytokines, chemokines, and growth factors, using a magnetic bead-based array and Luminex xMAP technology. Increases in the levels of cytokines with various immune activating and regulating properties were seen. This included a robust upregulation of certain proinflammatory cytokines, including IL-1 $\beta$, IL-6, IL-17, and TNF- $\alpha$ (Figure 3 ). Increases were also seen for the cytokines IL-4, IL-7, IL-8, IL-9, and IL-12p70 (data not shown). Furthermore, increases were seen for four biomarkers involved in antiviral immune defense activity, namely IFN- $\gamma$, and the three chemokines MCP-1, MIP-1 $\alpha$, and MIP-1 $\beta$ (Figure 4).

\section{Anti-inflammatory cytokines}

In parallel to increases in immune-activating, proinflammatory cytokines, higher doses of the inactivated B. coagulans GBI-30 cells also triggered robust increases in the two antiinflammatory cytokines IL-1ra and IL-10 (Figure 5). The increases were comparable between these cytokines, with an approximal 300-fold increase above the levels in untreated cell cultures.

\section{Growth factors}

The exposure of human PBMC to inactivated GBI-30 triggered changes in growth factor production (Figure 6). Highly selective changes were seen for the growth factors granulocyte-colony stimulating factor (G-CSF) and granulocyte macrophage-colony stimulating factor (GM-CSF), both having differential effects on stem cell biology (Figure 6A and $\mathrm{B}$ ), where treatment of PBMC with inactivated GBI-30 led to a very strong increase in G-CSF production, in contrast to a mild reduction in GM-CSF production.

\section{Discussion}

The work reported here on the inactivated B. coagulans GBI-30, 6086 cells is a direct extension of previous work by our team, involving the live B. coagulans GBI-30, 6086. Our previous results have demonstrated that the cell wall from $\mathrm{BC} 30$ has complex biological properties, including both immune-activating and anti-inflammatory properties. ${ }^{27}$

The current work showed that the inactivated $B$. coagulans GBI-30 cells triggered an increase in the CD69 activation marker on multiple human immune cell types, whereas previous work focused only on NK cells. CD69 is an early activation marker on many cell types, and is directly involved in the molecular apparatus responsible for NK cell-mediated killing of virus-infected and transformed cells. ${ }^{34-36}$ In addition, CD69 has a broad range of functions, including T-cell/B-cell interactions, homing of cells into appropriate tissue environments, and the process of generating and maintaining immunological memory. ${ }^{37,38}$ The ability of inactivated GBI-30 to induce CD69 on multiple cell types suggests a broad effect involving cells from both the innate and the adaptive immune systems. Further work will need to evaluate the various cell types in the non- $T$ non-NK subset of cells, which includes dendritic cells, B lymphocytes, circulating hematopoietic, mesenchymal, and endothelial stem cells. Previous work on live BC30 showed that exposure of human mononuclear phagocytes triggered a favorable maturation of antigen-presenting cells toward both macrophage and dendritic cell phenotypes. ${ }^{28}$ This is important, since dendritic cells are first-line antigenpresenting cells in gut mucosal immune tissue, capable of unique surveillance activity and antigen recognition across intact epithelial barriers, ${ }^{39}$ and therefore may represent the cell type that initially encounters consumed BC30 cells in the gut mucosa in vivo. 


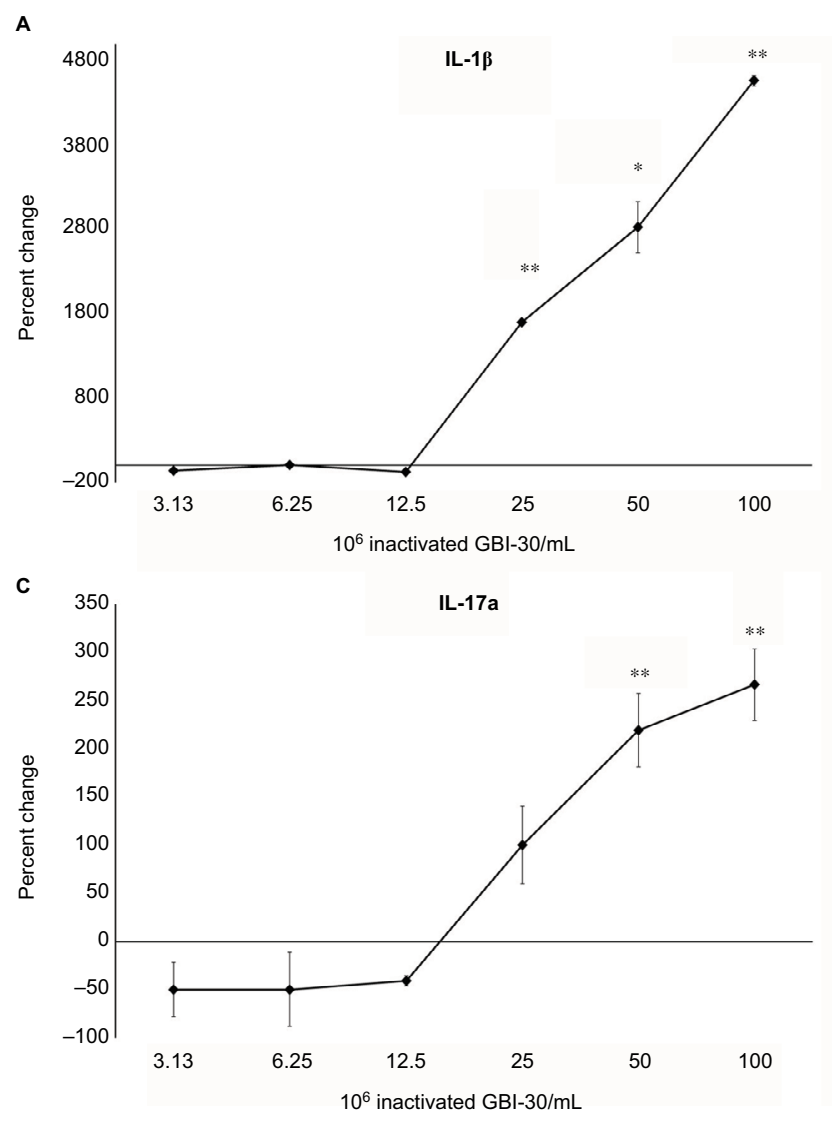

B

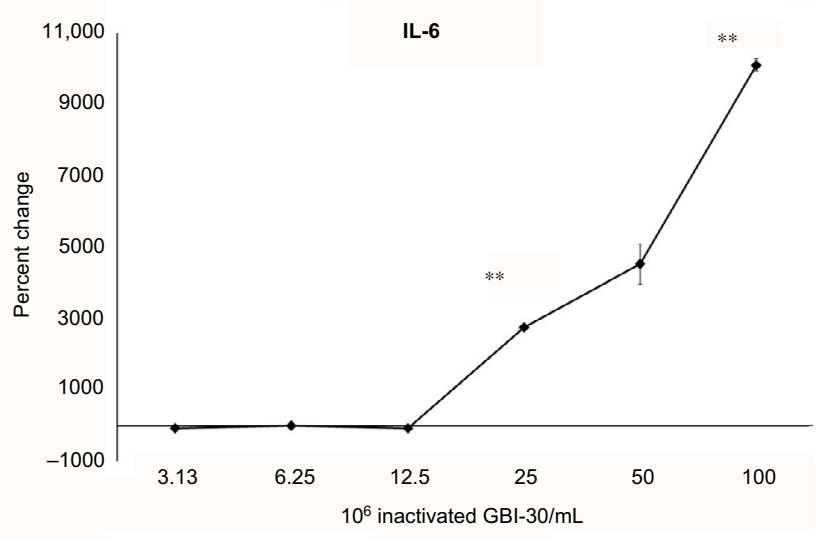

D

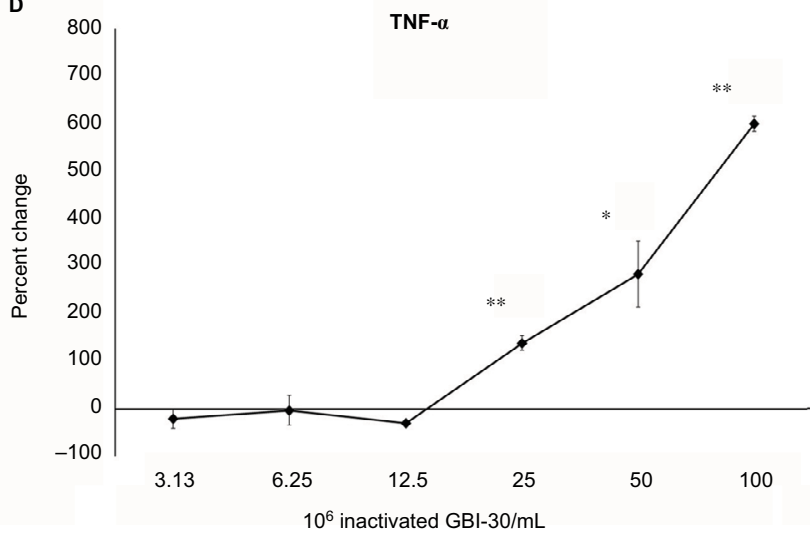

Figure 3 Changes in proinflammatory cytokine levels in human PBMC cultures.

Notes: ${ }^{*}<0.05 ; * * P<0.01$. Changes in cytokine levels in human PBMC cultures treated for 24 hours with serial dilutions of inactivated Bacillus coagulans GBI-30 cells are shown as percent change from untreated cell cultures. Data presented as mean \pm SD from duplicate testing of culture supernatants from one of three separate experiments using PBMC from three different healthy human donors.

Abbreviation: PBMC, peripheral blood mononuclear cells.

Inactivated B. coagulans GBI-30 cells triggered robust increases in the production of multiple cytokines, chemokines, and growth factors. The current data span a broader range of cytokines, chemokines, and growth factors than previously tested for live BC30. This has helped confirm the potent immune-activating properties of $\mathrm{BC} 30$. It has also helped demonstrate novel biological properties associated with antiviral and regenerative functions. The increases in the biomarkers IFN- $\gamma$, MCP-1, MIP- $1 \alpha$, and MIP- $1 \beta$, involved in antiviral immune defense mechanisms and cellular recruitment, are of special importance. IFN- $\gamma$ has direct antiviral properties, activates macrophages, and enhances NK cell killing activity of transformed cells. The three chemokines facilitate recruitment of immune cells to sites of inflammation, whether caused by injury or by infection. The increase in two anti-inflammatory cytokines, IL-1 ra and IL-10, points to the complexity of inactivated GBI-30 immune modulation. We speculate that the anti-inflammatory effect represents a later part of the cascade triggered by inactivated GBI-30, with the purpose of resolving the initial proinflammatory immune activation and limiting the inflammatory process in space and time.

Several growth factors known to play specific roles in endogenous regeneration were upregulated in the PBMC cultures. Highly selective, contrasting effects were seen for the two stem cell growth factors G-CSF and GM-CSF. Inactivated B. coagulans GBI-30-treated PBMC cultures showed a robust increase in G-CSF levels, reaching over 7,000-fold above untreated cultures at the highest dose, in contrast to mildly reduced GM-CSF levels. This is an important differentiation, since G-CSF supports stem cells to produce neutrophils, whereas GM-CSF promotes the production of multiple cell types, including eosinophils, involved in immune defense against multicellular parasites and also in inflammation in allergies and asthma. In addition, G-CSF is used therapeutically to support stem cell mobilization, migration, and tissue repair. ${ }^{40}$ Therefore, the selective 
A

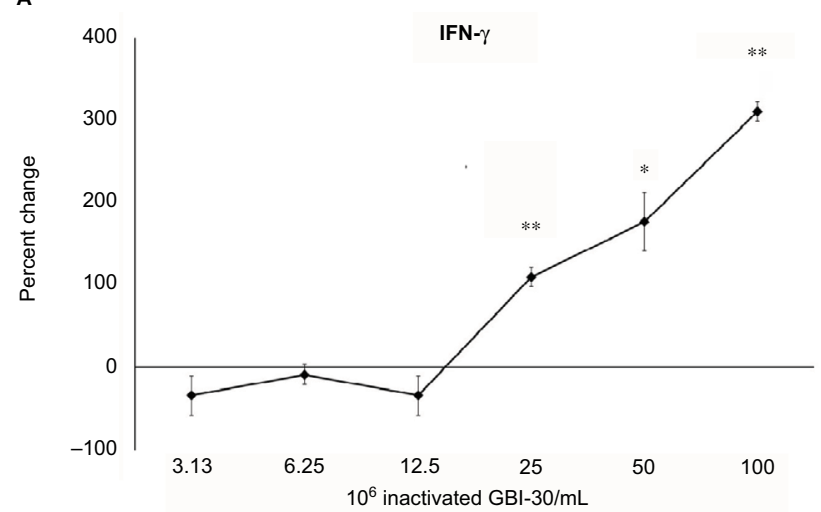

C

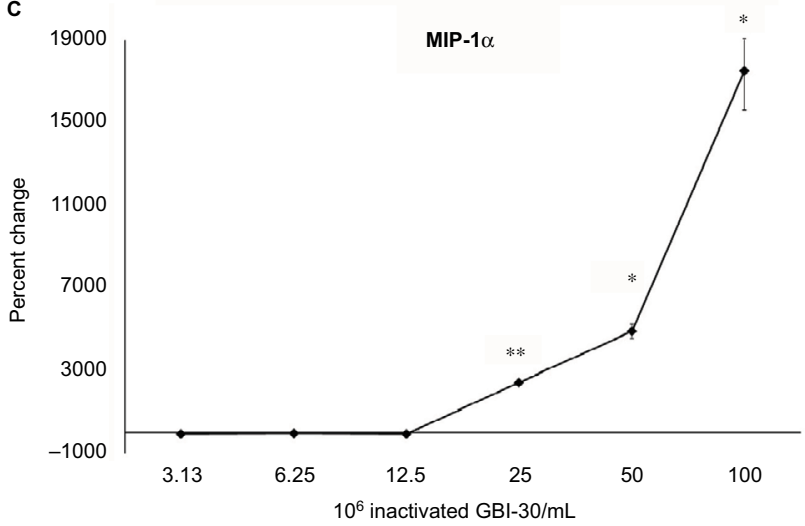

B
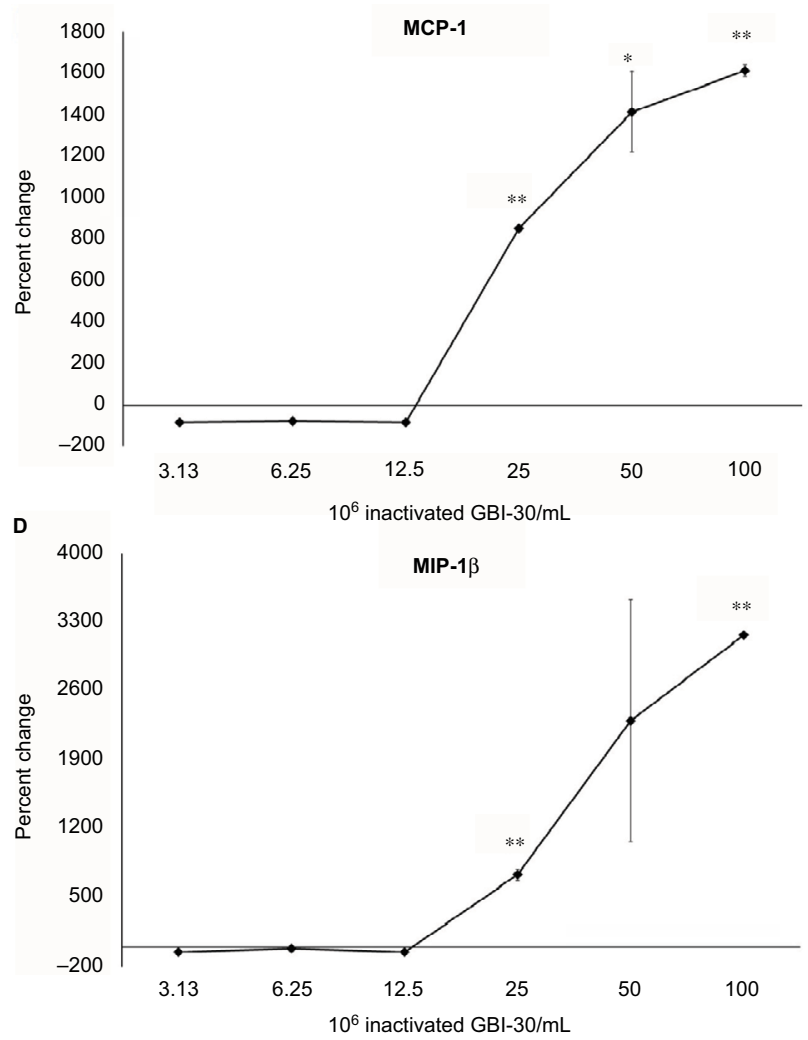

Figure 4 Changes in levels of cytokines involved in antiviral immunity in human PBMC cultures.

Notes: ${ }^{* P}<0.05 ;{ }^{*} * P<0.01$. Changes in cytokine levels in human PBMC cultures treated for 24 hours with serial dilutions of inactivated Bacillus coagulans GBI-30 cells are shown as percent change from untreated cell cultures. Data presented as mean \pm SD from duplicate supernatants from one of three separate experiments using PBMC from three different healthy human donors.

Abbreviation: PBMC, peripheral blood mononuclear cells.

effect of inactivated B. coagulans GBI-30 cells on growth factor production may be directly beneficial in repair and regeneration of the gut mucosal tissue, for example in situations of ulceration. The effect may also affect systemic changes via many cell types, including inflammation-modulating mesenchymal stem cells. ${ }^{41,42}$ Mesenchymal stem cells are able to sense signals from, and migrate into injured, inflamed, and ischemic tissue. They can cross the blood-brain barrier and contribute to repair of brain injuries such as stroke. Interestingly, it has been shown that the treatment of mesenchymal stem cells from healthy human donors with IL-1, TNF- $\alpha$, and IFN- $\gamma$ production contributed to a strong increase in G-CSF production by the mesenchymal stem cells ${ }^{43}$ and subsequently the increase in G-CSF reprogrammed LPSactivated microglial cells to secrete fewer inflammatory mediators. This sequential process may apply to events when mononuclear cells, which include multiple types of stem cells, are exposed to inactivated B. coagulans GBI-
30 cells. Further work is needed to document whether a cascade of events is triggered by inactivated GBI-30, where an initial immune-activating signal upregulated the production of proinflammatory cytokines, followed by anti-inflammatory processes intended to resolve the inflammation, combined with reparative growth factors.

Three observations from the current work reported here are of key importance in the validation of similar biological activities of the inactivated $B$. coagulans GBI-30 cells, compared to the immune-modulating properties of cell wall fractions from living BC30: 1) Both can increase the CD69 activation marker on lymphocytes, 2) both are able to increase production of the proinflammatory cytokine IL-6, and 3) both are capable of increasing the anti-inflammatory cytokine IL-10. This suggests that cell wall components, including lipoteichoic acid, have remained at least partially preserved by the inactivation process.

A direct dose comparison to our previous in vitro work is not feasible. Previous work on the cell wall's immune- 


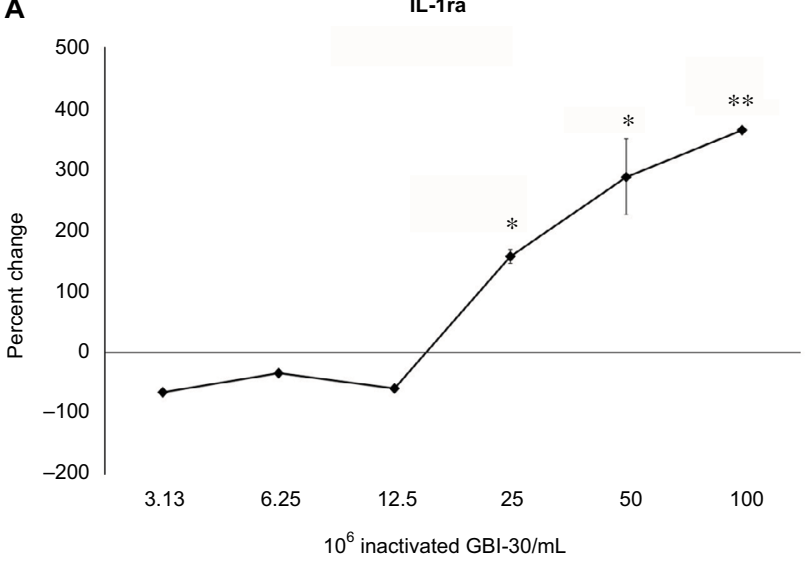

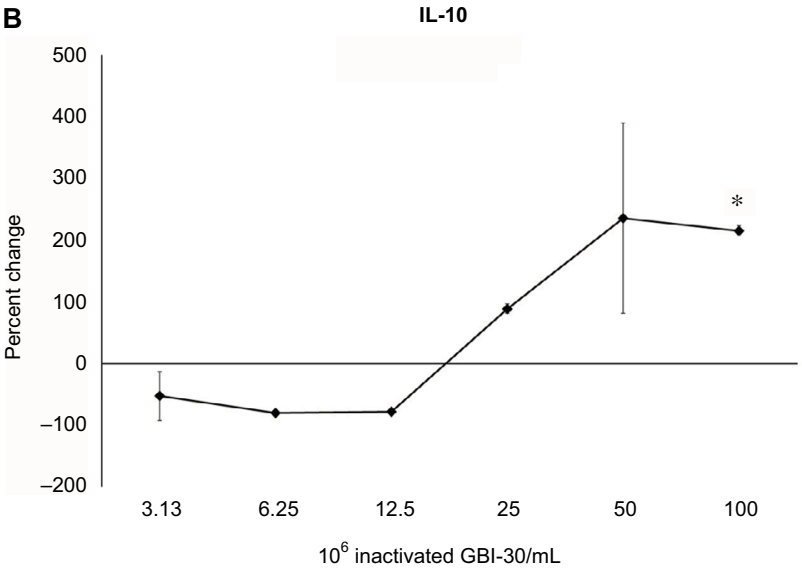

Figure 5 Changes in anti-inflammatory cytokine levels in human PBMC cultures treated for 24 hours.

Notes: $* P<0.05 ; * * P<0.0$ I. The first sentence should read: Changes in IL-I ra $(\mathbf{A})$ and IL-I0 (B) cytokine levels in human PBMC cultures treated for 24 hours with serial dilutions of inactivated Bacillus coagulans GBI-30 cells are shown as percent change from untreated cell cultures. Data presented as mean \pm SD from duplicate supernatants from one of three separate experiments using PBMC from three different healthy human donors.

Abbreviation: PBMC, peripheral blood mononuclear cells.
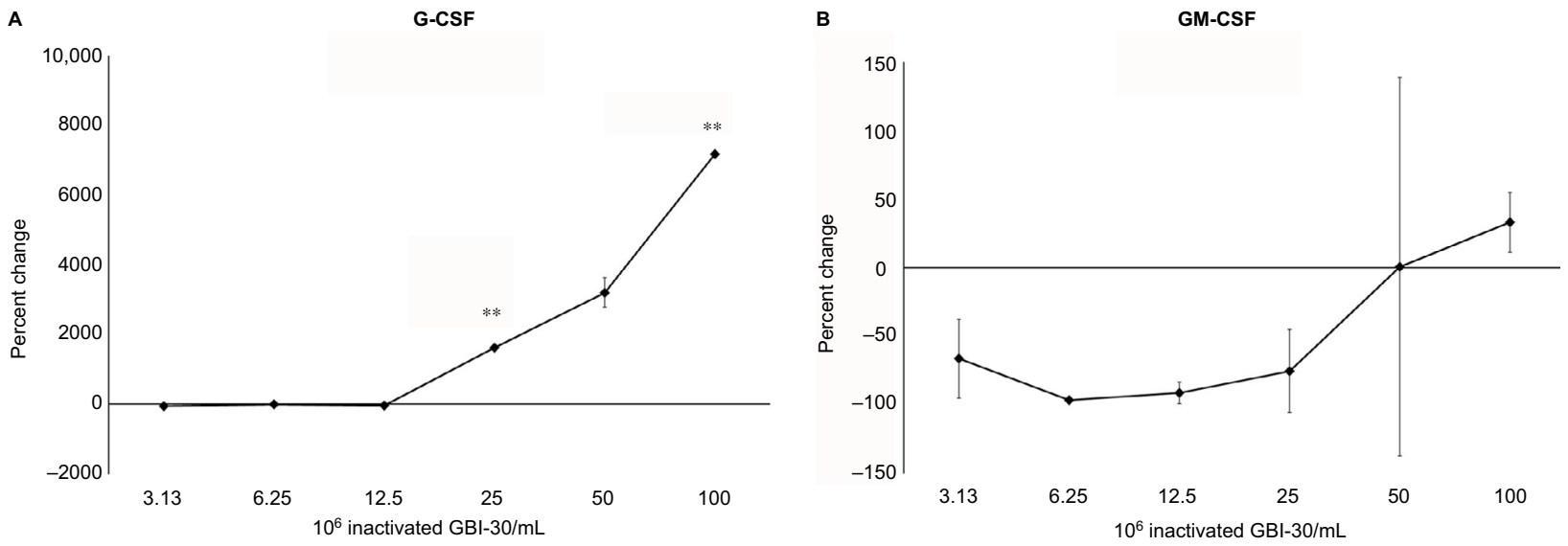

Figure 6 Changes in growth factor levels in human PBMC cultures treated for 24 hours.

Notes: $* * P<0.01$. Changes in G-CSF (A) and GM-CSF (B) growth factor levels in human PBMC cultures treated for 24 hours with serial dilutions of inactivated Bacillus coagulans GBI-30 cells are shown as percent change from untreated cell cultures. Data presented as mean \pm SD from duplicate supernatants from one of three separate experiments using PBMC from three different healthy human donors.

Abbreviations: G-CSF, granulocyte-colony stimulating factor; GM-CSF, granulocyte macrophage-colony stimulating factor; PBMC, peripheral blood mononuclear cells.

activating properties was performed on material that went through repeated freeze/thaw and bead-milling cycles, thus breaking down the cell walls into smaller particles, each capable of engaging appropriate receptors on the surface of the PBMC. In contrast, the current work involved the addition of intact bacterial cells to the human immune cell cultures. ${ }^{27}$

The complex properties of inactivated GBI-30 suggest possible multifaceted clinical responses after consumption, involving immune activation, anti-inflammatory effects, and effects involving stem cell mobilization, homing, and repro- gramming involved in accelerated repair. The direct effects of inactivated GBI-30 are expected to translate to immune activation at the level of the gut mucosa and trigger rapid systemic effects. This is different from a study on the live $\mathrm{BC} 30$ where ingested spores will give rise to living bacteria that can colonize the intestinal tract, and where an important part of the biological effects is due to secreted bacterial metabolites. Future work on inactivated $B$. coagulans GBI-30 cells should include a human clinical study to examine acute effects, using the study design previously published by our team on efficacious immune-modulating natural products, ${ }^{44,45}$ 
as well as nutraceutical products that have effects on human stem cell biology. ${ }^{46,47}$

\section{Acknowledgments}

The study was conducted at NIS Labs, an independent contract research laboratory specializing in the research of natural products. The study was sponsored by Ganeden Inc.

\section{Author contributions}

All authors contributed toward data analysis, drafting and critically revising the paper and agree to be accountable for all aspects of the work.

\section{Disclosure}

$\mathrm{HAC}, \mathrm{SF}$, and DK are employees of the sponsor of the study, Ganeden Inc. SF has also been a consultant for Ganeden Inc. GSJ reports no conflicts of interest in this work.

\section{References}

1. The Human Microbiome Project Consortium. Structure, function and diversity of the healthy human microbiome. Nature. 2012;486(7402):207-214.

2. Shreiner AB, Kao JK, Young VB. The gut microbiome in health and in disease. Curr Opin Gastroenterol. 2015;31(1):69-75.

3. Galland L. The gut microbiome and the brain. J Med Food. 2014;17(12): 1261-1272.

4. Gioannini TL, Weiss JP. Regulation of interactions of Gram-negative bacterial endotoxins with mammalian cells. Immunol Res. 2007; 39(1-3):249-260.

5. Schwandner R, Dziarski R, Wesche H, Rothe M, Kirschning CJ. Peptidoglycan- and lipoteichoic acid-induced cell activation is mediated by toll-like receptor 2. J Biol Chem. 1999;274(25):17406-17409.

6. Kang SS, Sim JR, Yun CH, Han SH. Lipoteichoic acids as a major virulence factor causing inflammatory responses via toll-like receptor 2. Arch Pharm Res. 2016;39(11):1519-1529.

7. Paustian C, Taylor P, Johnson T, et al. Extracellular ATP and toll-like receptor 2 agonists trigger in human monocytes an activation program that favors T helper 17. PLoS One. 2013;8(1):e54804.

8. Cheng SF, Ho JW, Chan KY, et al. IL-15 and macrophage secretory factors facilitate immune activation of neonatal natural killer cells by lipoteichoic acid. Cytokine. 2013;61(2):499-505.

9. Kim H, Jung BJ, Jung JH, Kim JY, Chung SK, Chung DK. Lactobacillus plantarum lipoteichoic acid alleviates TNF- $\alpha$-induced inflammation in the HT-29 intestinal epithelial cell line. Mol Cells. 2012;33(5):479-486.

10. Kim HG, Lee SY, Kim NR, et al. Lactobacillus plantarum lipoteichoic acid down-regulated Shigella flexneri peptidoglycan-induced inflammation. Mol Immunol. 2011;48(4):382-391.

11. Korpela K, Salonen A, Virta LJ, Kumpu M, Kekkonen RA, de Vos WM. Lactobacillus rhamnosus GG intake modifies preschool children's intestinal microbiota, alleviates penicillin-associated changes, and reduces antibiotic use. PLoS One. 2016;11(4):e0154012.

12. Lenoir-Wijnkoop I, Gerlier L, Roy D, Reid G. The clinical and economic impact of probiotics consumption on respiratory tract infections: projections for Canada. PLoS One. 2016;11(11):e0166232.

13. Wang Y, Kasper LH. The role of microbiome in central nervous system disorders. Brain Behav Immun. 2014;38:1-12.

14. Sanchez M, Darimont C, Panahi S, et al. Effects of a diet-based weightreducing program with probiotic supplementation on satiety efficiency, eating behaviour traits, and psychosocial behaviours in obese individuals. Nutrients. 2017;9(3):E284.
15. Wallace CJK, Milev R. The effects of probiotics on depressive symptoms in humans: a systematic review. Ann Gen Psychiatry. 2017;16:14.

16. Dinan TG, Cryan JF. The microbiome-gut-brain axis in health and disease. Gastroenterol Clin North Am. 2017;46(1):77-89.

17. Maeda N, Nakamura R, Hirose Y, et al. Oral administration of heat-killed Lactobacillus plantarum L-137 enhances protection against influenza virus infection by stimulation of type I interferon production in mice. Int Immunopharmacol. 2009;9(9):1122-1125.

18. Hirose Y, Murosaki S, Yamamoto Y, Yoshikai Y, Tsuru T. Daily intake of heat-killed Lactobacillus plantarum L-137 augments acquired immunity in healthy adults. J Nutr. 2006;136(12):3069-3073.

19. Hirose Y, Yamamoto Y, Yoshikai Y, Murosaki S. Oral intake of heat-killed Lactobacillus plantarum L-137 decreases the incidence of upper respiratory tract infection in healthy subjects with high levels of psychological stress. J Nutr Sci. 2013;2:e39.

20. Iwasaki K, Maeda K, Hidaka K, Nemoto K, Hirose Y, Deguchi S. Daily intake of heat-killed Lactobacillus plantarum L-137 decreases the probing depth in patients undergoing supportive periodontal therapy. Oral Health Prev Dent. 2016;14(3):207-214.

21. Murosaki S, Yamamoto Y, Ito K, et al. Heat-killed Lactobacillus plantarum L-137 suppresses naturally fed antigen-specific IgE production by stimulation of IL-12 production in mice. J Allergy Clin Immunol. 1998;102(1):57-64.

22. Guo B, Xie N, Wang Y. Cooperative effect of bifidobacteria lipoteichoic acid combined with 5-fluorouracil on hepatoma-22 cells growth and apoptosis. Bull Cancer. 2015;102(3):204-212.

23. Arimori Y, Nakamura R, Hirose Y, et al. Daily intake of heat-killed Lactobacillus plantarum L-137 enhances type I interferon production in healthy humans and pigs. Immunopharmacol Immunotoxicol. 2012;34(6): 937-943.

24. Hatano S, Hirose Y, Yamamoto Y, Murosaki S, Yoshikai Y. Scavenger receptor for lipoteichoic acid is involved in the potent ability of Lactobacillus plantarum strain L-137 to stimulate production of interleukin12p40. Int Immunopharmacol. 2015;25(2):321-331.

25. Hirose Y, Murosaki S, Fujiki T, Yamamoto Y, Yoshikai Y, Yamashita M. Lipoteichoic acids on Lactobacillus plantarum cell surfaces correlate with induction of interleukin-12p40 production. Microbiol Immunol. 2010;54(3):143-151

26. Forrester, IT, Wicken, AJ. The chemical composition of the cell walls of some thermophilic bacilli. J Gen Microbiol. 1966;42(1):147-154

27. Jensen GS, Benson KF, Carter SG, Endres JR. GanedenBC30 cell wall and metabolites: anti-inflammatory and immune modulating effects in vitro. BMC Immunol. 2010;11:15

28. Benson KF, Redman KA, Carter SG, et al. Probiotic metabolites from Bacillus coagulans GanedenBC $30^{\mathrm{TM}}$ support maturation of antigen-presenting cells in vitro. World J Gastroenterol. 2012;18(16):1875-1883.

29. Fitzpatrick LR, Small JS, Greene WH, Karpa KD, Keller D. Bacillus coagulans GBI-30 (BC30) improves indices of clostridium difficileinduced colitis in mice. Gut Pathog. 2011;3(1):16.

30. Fitzpatrick LR, Small JS, Greene WH, Karpa KD, Farmer S, Keller D. Bacillus coagulans GBI-30, 6086 limits the recurrence of clostridium difficile-induced colitis following vancomycin withdrawal in mice. Gut Pathog. 2012;4(1):13.

31. Nyangale EP, Farmer S, Keller D, Chernoff D, Gibson GR. Effect of prebiotics on the fecal microbiota of elderly volunteers after dietary supplementation of Bacillus coagulans GBI-30, 6086. Anaerobe. 2014;30: 75-81.

32. Nyangale EP, Farmer S, Cash HA, Keller D, Chernoff D, Gibson GR. Bacillus coagulans GBI-30, 6086 modulates faecalibacterium prausnitzii in older men and women. J Nutr. 2015;145(7):1446-1452.

33. Jäger R, Shields KA, Lowery RP, et al. Probiotic Bacillus coagulans GBI-30, 6086 reduces exercise-induced muscle damage and increases recovery. Peer J. 2016;4:e2276.

34. Moretta A, Poggi A, Pende D, et al. CD69-mediated pathway of lymphocyte activation: anti-CD69 monoclonal antibodies trigger the cytolytic activity of different lymphoid effector cells with the exception of cytolytic T lymphocytes expressing T cell receptor alpha/beta. J Exp Med. 1991;174(6):1393-1398. 
35. Dons'koi BV, Chernyshov VP, Osypchuk DV. Measurement of NK activity in whole blood by the CD69 up-regulation after co-incubation with K562, comparison with NK cytotoxicity assays and CD107a degranulation assay. J Immunol Methods. 2011;372(1-2):187-195.

36. Clausen J, Vergeiner B, Enk M, Petzer AL, Gastl G, Gunsilius E. Functional significance of the activation-associated receptors CD25 and CD69 on human NK-cells and NK-like T-cells. Immunobiology. 2003;207(2): 85-93.

37. Schoenberger SP. CD69 guides CD4+ T cells to the seat of memory. Proc Natl Acad Sci U S A. 2012;109(22):8358-8359.

38. Cibrián D, Sánchez-Madrid F. CD69: from activation marker to metabolic gatekeeper. Eur J Immunol. 2017;47(6):946-953.

39. Allen F, Tong AA, Huang AY. Unique transcompartmental bridge: antigen-presenting cells sampling across endothelial and mucosal barriers. Front Immunol. 2016;7:231.

40. Chan SJ, Love C, Spector M, Cool SM, Nurcombe V, Lo EH. Endogenous regeneration: engineering growth factors for stroke. Neurochem Int. 2017;107:57-65.

41. Lee S, Zhang QZ, Karabucak B, Le AD. DPSCs from inflamed pulp modulate macrophage function via the TNF- $\alpha /$ IDO axis. J Dent Res. 2016;95(11):1274-1281.
42. Sica A, Mantovani A. Macrophage plasticity and polarization: in vivo veritas. J Clin Invest. 2012;122(3):787-795.

43. Redondo-Castro E, Cunningham C, Miller J, et al. Interleukin-1 primes human mesenchymal stem cells towards an anti-inflammatory and protrophic phenotype in vitro. Stem Cell Res Ther. 2017;8(1):79.

44. Jensen GS, Redman KA, Benson KF, et al. Antioxidant bioavailability and rapid immune-modulating effects after consumption of a single acute dose of a high-metabolite yeast immunogen: results of a placebocontrolled double-blinded crossover pilot study. J Med Food. 2011;14(9): 1002-1010.

45. Jensen GS, Patel D, Benson KF. A novel extract from bovine colostrum whey supports innate immune functions. II. Rapid changes in cellular immune function in humans. Prev Med. 2012;54 Suppl:S124-S129.

46. Jensen GS, Hart AN, Zaske LA, et al. Mobilization of human CD34+ CD133+ and CD34+ CD133(-) stem cells in vivo by consumption of an extract from aphanizomenon flos-aquae related to modulation of CXCR4 expression by an L-selectin ligand?. Cardiovasc Revasc Med. 2007;8(3):189-202.

47. Drapeau CJ, Benson KF, James J, Jensen G. Aloe macroclada from Madagascar Triggers Transient Bone Marrow Stem Cell Mobilization. J Stem Cell Res Ther. 2015;5:287.
Journal of Inflammation Research

\section{Publish your work in this journal}

The Journal of Inflammation Research is an international, peer-reviewed open access journal that welcomes laboratory and clinical findings on the molecular basis, cell biology and pharmacology of inflammation including original research, reviews, symposium reports, hypothesis formation and commentaries on: acute/chronic inflammation; mediators of

\section{Dovepress}

inflammation; cellular processes; molecular mechanisms; pharmacology and novel anti-inflammatory drugs; clinical conditions involving inflammation. The manuscript management system is completely online and includes a very quick and fair peer-review system. Visit http://www.dove press.com/testimonials.php to read real quotes from published authors. 